\title{
An Experimental and Theoretical Multi-Mbar Study of Ti-6Al-4V
}

B. E. Tegner, S. G. Macleod, H. CYNN, J. Proctor, W. J. Evans, M. I McMahon, G. J. Ackland

April 22, 2011

2011 MRS Spring Meeting

San Francisco, CA, United States

April 25, 2011 through April 29, 2011 
This document was prepared as an account of work sponsored by an agency of the United States government. Neither the United States government nor Lawrence Livermore National Security, LLC, nor any of their employees makes any warranty, expressed or implied, or assumes any legal liability or responsibility for the accuracy, completeness, or usefulness of any information, apparatus, product, or process disclosed, or represents that its use would not infringe privately owned rights. Reference herein to any specific commercial product, process, or service by trade name, trademark, manufacturer, or otherwise does not necessarily constitute or imply its endorsement, recommendation, or favoring by the United States government or Lawrence Livermore National Security, LLC. The views and opinions of authors expressed herein do not necessarily state or reflect those of the United States government or Lawrence Livermore National Security, LLC, and shall not be used for advertising or product endorsement purposes. 


\title{
An Experimental and Theoretical Multi-Mbar Study of Ti-6Al-4V
}

\author{
Bengt E. Tegner ${ }^{1}$, Simon G. MacLeod ${ }^{2}$, Hyunchae Cynn ${ }^{3}$, John Proctor $^{1}$, William J. Evans ${ }^{3}$, \\ Malcolm I. McMahon ${ }^{1}$ and Graeme J. Ackland ${ }^{1}$ \\ ${ }^{1}$ SUPA, School of Physics and Astronomy, and Centre for Science at Extreme Conditions. The \\ University of Edinburgh, EH9 3JZ, U.K. \\ ${ }^{2}$ Institute of Shock Physics, Imperial College London, SW7 2AZ, U.K. \\ ${ }^{3}$ Lawrence Livermore National Laboratories, Livermore, CA 94550, U.S.A.
}

\begin{abstract}
We report results from an experimental and theoretical study of the room temperature (RT) compression of the ternary alloy Ti-6Al-4V. In this work, we have extended knowledge of the equation of state (EOS) from $40 \mathrm{GPa}$ to $221 \mathrm{GPa}$, and observed a different sequence of phase transitions to that reported previously for pure Ti.
\end{abstract}

\section{INTRODUCTION}

The commercial and industrial importance of the two-phase ternary alloy Ti-6Al-4V (wt $\%$ ) is well documented and known to be heavily dependent on its mechanical properties (see for example [1]). Ti-6Al-4V (hereafter referred to as Ti64) crystallizes predominantly in the twoatom $\alpha$-phase (hexagonal-close-packed or hcp) at ambient conditions, with a much smaller fraction by volume crystallizing in the $\beta$-phase (body-centred-cubic or bcc) around the grain boundaries. The alloying of substitutional and interstitial impurities increases the strength of Ti64 compared with pure Ti. Al is the $\alpha$-phase stabilizer and dominant substitutional strengthener.

At room temperature (RT), using diamond anvil cells (DACs) and angle-dispersive X-ray diffraction (ADXRD) [2], the $\alpha$-phase of Ti64 has been observed to transform into the threeatom hexagonal $\omega$-phase at $27 \mathrm{GPa}$. A more recent RT DAC study, using energy-dispersive Xray diffraction (EDXRD), did not observe any phase transformation up to $32.4 \mathrm{GPa}$ [3]. Shock studies of Ti64 are fairly extensive, but no evidence of a phase transformation has been observed (see for example [4] and references contained therein).

Pure Ti has been compressed at RT using DACs to $220 \mathrm{GPa}$, and the transformation sequence $\alpha \rightarrow \omega \rightarrow \gamma \rightarrow \delta$ was reported [5,6], where the $\gamma$ and $\delta$ phases are orthorhombic distortions of the hep and bcc structures, respectively. The effects of uniaxial stress on the $\alpha \rightarrow \omega$ transition in Ti were studied by Errandonea et al. [7] using DACs and ADXRD. They found that the transition pressure was dependent on the pressure transmitting medium (PTM) used, and ranged from 4.9 GPa (no PTM) up to $10.5 \mathrm{GPa}$ (argon PTM). In addition, they observed the coexistence of the $\alpha$ and $\omega$ phases over a large pressure range.

Our motivation for conducting the present study was to determine whether or not Ti64 exhibited similar behavior to that reported for pure Ti at multi-megabar pressures. 


\section{EXPERIMENT AND THEORY}

\section{Experimental details}

We performed a number of static high-pressure compression experiments using gasmembrane driven DACs at the High Pressure Collaborative Access Team (HPCAT) beamline 16IDB, at the Advanced Photon Source (APS), in Chicago. We collected ADXRD patterns from commercially-sourced Ti64 powder (Goodfellow) embedded in a variety of pressure media, and loaded into rhenium gaskets with either a $\mathrm{Cu}$ or a Ta pressure marker.

Initially, we studied the $\alpha \rightarrow \omega$ phase transition. In order to investigate the effects of uniaxial stress on this transition we used a variety of PTMs, and loaded samples of Ti64 into DACs with (in increasing order of hydrostaticity): no PTM; mineral oil; 4:1 methanol-ethanol; and neon. Figure 1 shows a representative stacked plot of ADXRD patterns obtained from Ti64 embedded in a neon PTM and with a $\mathrm{Cu}$ pressure marker. We clearly observe a coexistence of the $\alpha$ and $\omega$ phases over a large pressure range (between $32.7 \mathrm{GPa}$ and $\sim 44.4 \mathrm{GPa}$ ). The dominant $\omega(110 / 101)$ reflections first appear at $\sim 32.7 \mathrm{GPa}$, and with further increases in pressure, the $\omega(110 / 101)$ reflections increase in intensity, the strong $\alpha(101)$ reflection decreases in intensity, and the weaker, $\omega(001)$ and $\omega$ (201) peaks appear. By $44.4 \mathrm{GPa}$, the $\alpha-$ phase peaks have almost completely disappeared. In all, we were able to index up to $11 \alpha$-phase peaks and $8 \omega$-phase peaks.

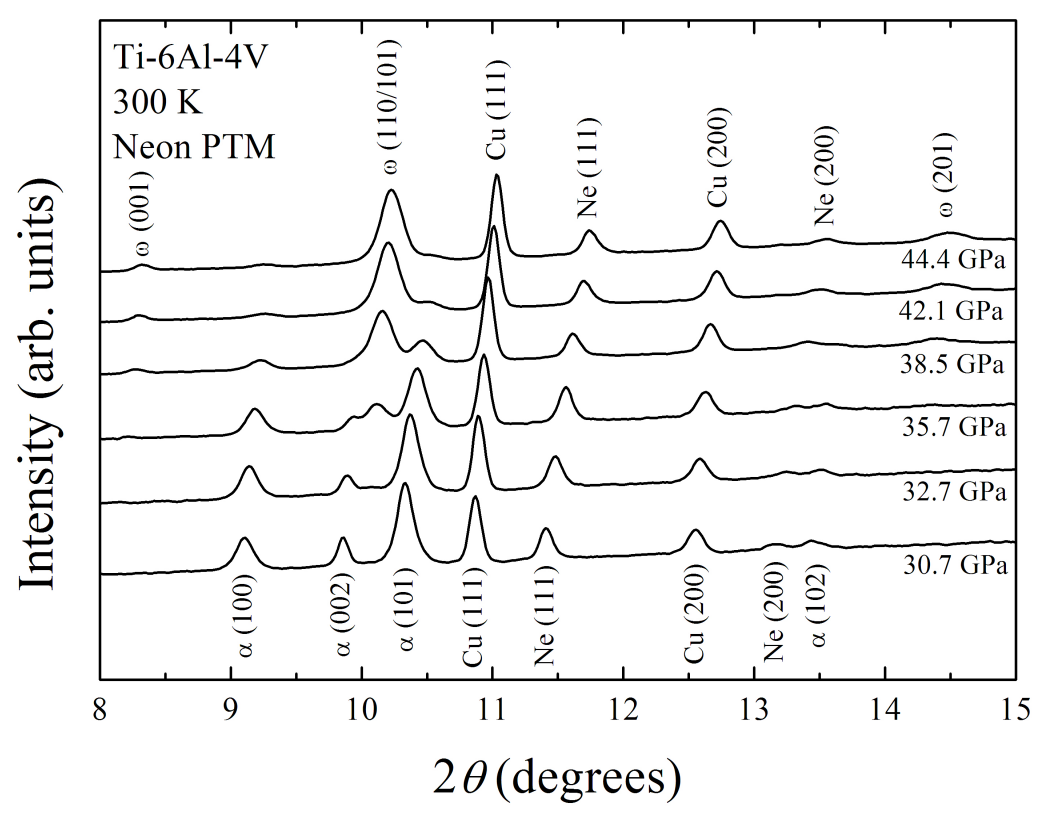

Figure 1. A stacked plot showing Ti64 ADXRD patterns collected on pressure increase from 30.7 GPa to $44.4 \mathrm{GPa}$. Diffraction peaks from the the $\alpha$ and $\omega$ phases, and from the $\mathrm{Cu}$ pressure marker and neon PTM are indexed.

We observed similar behavior in the $\alpha \rightarrow \omega$ transformation for all of the PTMs used in this study. The more hydrostatic the PTM, the higher the pressure at which the $\alpha \rightarrow \omega$ transition occurred. Table I summarizes the observed transition pressures, and the measured $K_{0}$ and $K^{\prime}$ of 
the $\alpha$-phase in different PTMs (including previously-published observations). The $\alpha$-phase data were fitted using the Vinet EOS formulation [8]. We measured the volume at ambient conditions to be $V_{0}=17.252 \AA^{3}$.

Table I. Summary of the observed $\alpha \rightarrow \omega$ transformation for Ti64. The transition pressure is the first appearance of peaks from the $\omega$ phase. $K_{0}$ and $K^{\prime}$ are the bulk modulus, and its pressure derivative, of the $\alpha$ phase, as determined from fitting the compression data to a Vinet EOS.

\begin{tabular}{llll}
\hline Pressure medium (PTM) & $P \alpha \rightarrow \omega(\mathrm{GPa})$ & $K_{0}(\mathrm{GPa})$ & $K^{\prime}$ \\
\hline No medium & 26.4 & $100 \pm 12$ & $4.46 \pm 1.50$ \\
Mineral oil & 26.2 & $106 \pm 10$ & $5.07 \pm 1.23$ \\
4:1 methanol-ethanol & 31.2 & $115 \pm 3$ & $3.22 \pm 0.22$ \\
Neon & 32.7 & $101 \pm 3$ & $4.05 \pm 0.29$ \\
4:1 methanol-ethanol [2] & 27.3 & 125 & 2.41 \\
N/A [3] & N/A & $154 \pm 11$ & $5.45 \pm 1.44$ \\
\hline
\end{tabular}

To search for further phase transitions at higher pressures, we compressed Ti64 in a mineral oil PTM to $221 \mathrm{GPa}$. We observed a sluggish phase transition between $95 \mathrm{GPa}$ and 123 GPa to a new phase (henceforth referred to as the $\beta$ phase) which had the bcc structure. Figure 2 shows integrated ADXRD patterns obtained over this pressure range, showing the gradual disappearance of the (210) and (002) reflections from the $\omega$ phase that mark this transition.

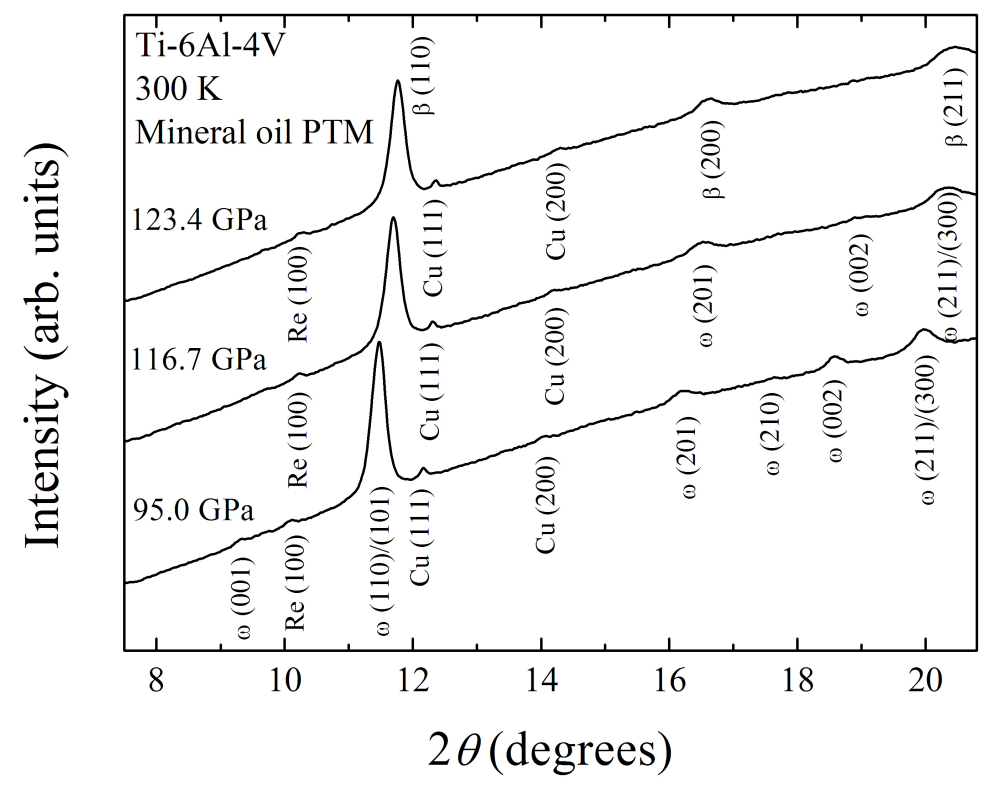

Figure 2. ADXRD patterns of the $\omega \rightarrow \beta$ phase transition for Ti64 between 95 and $123 \mathrm{GPa}$. Peaks from the $\omega$ and $\beta$ phases, the $\mathrm{Cu}$ pressure marker and the Re gasket are indexed. 
The $\beta$-phase is stable to $221 \mathrm{GPa}$, the highest pressure reached in this study, and we find no evidence of the orthorhombic $\gamma$ and $\delta$ phases reported in pure Ti. The $P-V$ plot of the RT compression of Ti64 in a mineral oil PTM to $221 \mathrm{GPa}$ is shown in Figure 3.

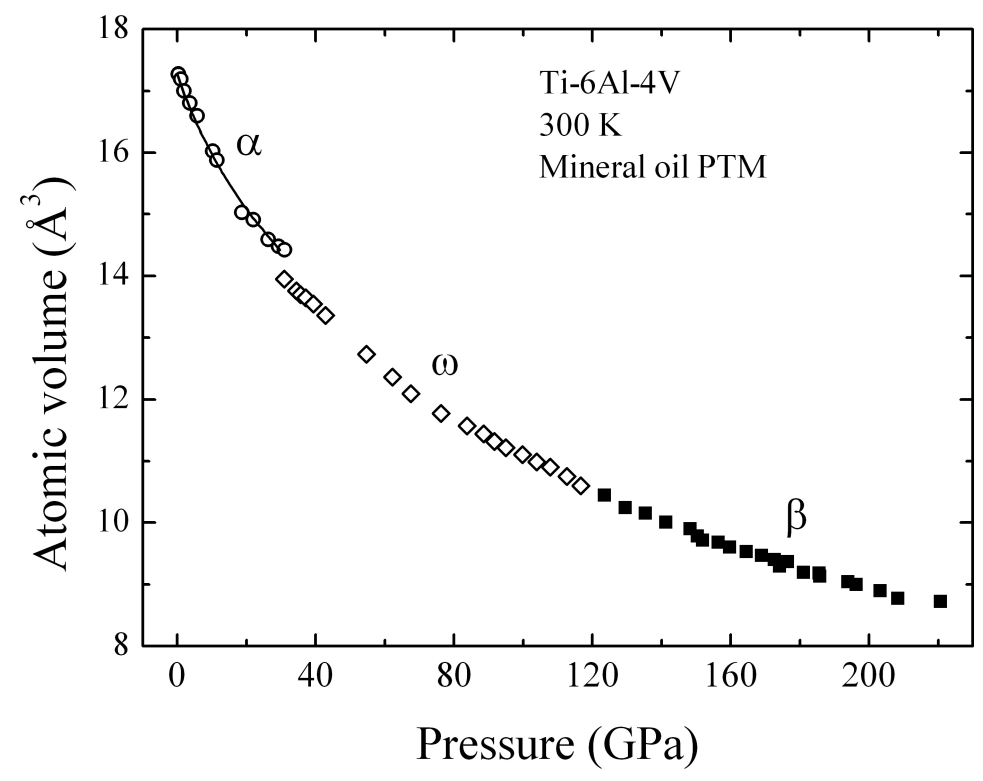

Figure 3. The change in the atomic volume and crystal structure of Ti64 with increasing pressure. The solid line shows the Vinet fit to the $\alpha$-phase data.

The volume change across the $\alpha-\omega$ boundary was less than $1 \%$ for Ti64 in both a neon and 4:1 methanol-ethanol PTM. For Ti64 in mineral oil, the volume change was $2.5 \%$ and for no PTM the volume change was $4.5 \%$. However, the Ti64 data collected using no PTM was difficult to analyze because of the presence of Re peaks in the diffraction patterns at pressures below $30 \mathrm{GPa}$. Consequently, our quoted volume change across the $\alpha-\omega$ boundary may be in error.

\section{$\underline{\text { Theoretical Details }}$}

We performed our theoretical calculations using the plane-wave DFT-code CASTEP [9], using supercells of 54 atoms for each configuration, to get impurity levels of $2 \mathrm{at} \%$. The k-point density for each configuration was $0.05 \AA^{-1}$. A plane-wave cut-off of $500 \mathrm{eV}$ was used for basisset convergence. In all cases, the alloy composition was 85.2 at $\% \mathrm{Ti}, 11.1$ at $\% \mathrm{Al}$ and 3.7 at $\% \mathrm{~V}$, that is, 46 atoms of Ti, 6 atoms of $\mathrm{Al}$ and 2 atoms of $\mathrm{V}$. We chose the one that gave the lowest energy.

The calculated enthalpies of the $\omega$ and $\beta$ phases, relative to that of the $\alpha$-phase, (at $\mathrm{T}=0$ $\mathrm{K}$ ) as a function of pressure are shown in Figure 4. The calculated $\alpha \rightarrow \omega$ transition pressure is 
$24 \mathrm{GPa}$, in agreement with our experimental data. The transition pressure to the $\beta$ phase is predicted to be around $110 \mathrm{GPa}$, again in reasonable agreement with our experimental data.

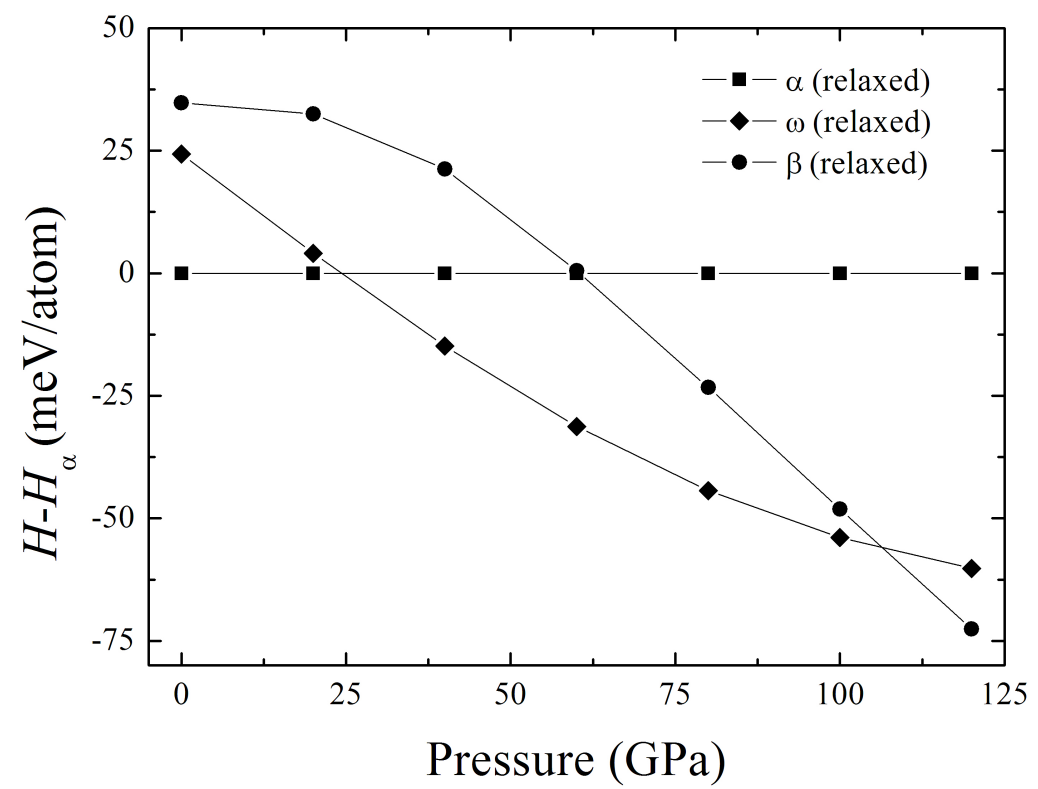

Figure 4. The calculated enthalpies of the $\omega$ and $\beta$ phases, relative to that of the $\alpha$-phase, as a function of increasing pressure. The transition pressures are taken from where the lower curves cross.

Above $110 \mathrm{GPa}$, our calculations found the cubic $\beta$ phase to always be more stable than the orthorhombic $\gamma$ and $\delta$ phases.

\section{DISCUSSION AND CONCLUSIONS}

We have performed a series of ADXRD DAC experiments to extend knowledge of the EOS of Ti64 from $40 \mathrm{GPa}$ to $221 \mathrm{GPa}$. In so doing, we have confirmed the occurrence of the $\alpha \rightarrow \omega$ phase transition at a much higher pressure $(26.2 \mathrm{GPa}-32.7 \mathrm{GPa})$ than is the case for pure $\mathrm{Ti}$ ( $4.9 \mathrm{GPa}-10.5 \mathrm{GPa}$ ). This is to be expected, as the alloying of the $\alpha$-phase stabilizer (Al) suppresses the $\alpha \rightarrow \omega$ phase transition.

In contrast to a previous study [2], we observed the coexistence of the $\alpha$ and $\omega$ phases over a large pressure range. Errandonea et al. also observed such a coexistence of these phases for pure $\mathrm{Ti}$ [7]. Thermodynamically, it should not be possible to have a coexistence of phases over a range of hydrostatic pressures without chemical separation. However, the observation of phase coexistence is not uncommon in DAC experiments and could well be a consequence of the non-hydrostatic conditions that inevitably exist in this uniaxial pressure device, even when using a PTM such as neon.

We observed a phase transition from the $\omega$ phase to the bcc $\beta$-phase between $117 \mathrm{GPa}$ and $123 \mathrm{GPa}$ when using a mineral oil PTM, and found the $\beta$-phase to be stable to at least 221 $\mathrm{GPa}$. This transition sequence is different to the reported for pure $\mathrm{Ti}$, where there are transitions 
to the orthorhombic $\delta$ and $\gamma$ phases [5, 6]. A possible cause of the different transition sequence found in Ti64 could be the presence of a PTM in our experiment, as opposed to the absence of a PTM in the previous Ti experiments [5, 6]. Studies of Ti to above $200 \mathrm{GPa}$ using a PTM are planned. DFT calculations of the $\alpha \rightarrow \omega$ and $\omega \rightarrow \beta$ transition pressures are in good agreement with our observed values.

\section{ACKNOWLEDGMENTS}

Theoretical work is supported by the European project MaMiNa, which aims to develop new, more easily machinable, titanium alloys, looking at macro, micro and nano aspects. HPCAT is supported by CIW, CDAC, UNLV and LLNL through funding from DOE-NNSA, DOEBES and NSF. APS is supported by DOE-BES, under Contract No. DE-AC02-06CH11357. This work was performed under the auspices of the U.S. Department of Energy by Lawrence Livermore National Laboratory in part under Contract W-7405-Eng-48 and in part under Contract DE-AC52-07NA27344

\section{REFERENCES}

1. M. Peters, J. Hemptenmacher, J. Kumpfert, and C. Leyens, in Titanium and Titanium Alloys: Fundamentals and Applications, edited by C. Leyens and M. Peters (Wiley-VCH, Weinheim, Germany, 2003) pp. 1-35.

2. G. N. Chesnut, N. Velisavljevic, and L. Sanchez in Shock Compression of Condensed Matter - 2007, edited by M. Elert, M. D. Furnish, R. Chau, N. Holmes, and J, Nguyen, (American Institute of Physics, New York, 2007) pp. 27-30.

3. I. Halevy, G. Zamir, M. Winterrose, G. Sanjit, C. R. Grandini, and A. Moreno-Gobbi, J. Phys.: Conf. Ser. 215, 012013 (2010).

4. N. K. Bourne, J. C. F. Millett, and G. T. Gray III, J. Mater. Sci. 44, 3319 (2009).

5. Y. K. Vohra and P. T. Spencer, Phys. Rev. Lett. 86, 3068 (2001).

6. Y. Akahama, H. Kawamura, and T. Le Bihan, Phys. Rev. Lett. 87, 275503 (2001).

7. D. Errandonea, Y. Meng, M. Somayazulu, and D. Häusermann, Physica B 355, 116 (2005).

8. P. Vinet, J. Ferrante, J. Rose, and J. Smith, J. Geophys. Res. 92, 9319 (1987).

9. S. J. Clark, M. D. Segall, C. J. Pickard, P. J. Hasnip, M. I. J. Probert, K. Refson, and M. C. Payne, Z. Kristallogr. 220, 567 (2005). 